\title{
Designing products and services based on understanding human cognitive behavior
}

— Development of cognitive chrono-ethnography for synthesiological research —

\author{
Motoyuki AKamatsu ${ }^{1 *}$ and Muneo KITAJIMA ${ }^{2}$
}

[Translation from Synthesiology, Vol.4, No.3, p.140-150 (2011)]

\begin{abstract}
When technology development is conducted based only on a developer's idea, we face difficulties in realizing products and services that will be well accepted by people. This is because it is difficult for a developer to know precisely how various users think and feel while using products or receiving services under real life situations. In order to overcome these difficulties, we have developed cognitive chronoethnography (CCE) which is a new method to understand cognitive behavior under various situations. This method clarifies the cognitive behavior process by selecting "elite monitors" taking characteristics of target users into consideration, identifying "critical parameters" utilized in designing the products or services, recording the human behavior in real life situations under the control of the parameters, and retrospectively interviewing the participants. This method is applied in the early stages of synthesiological R\&D processes oriented toward designing target products and services acceptable by people.
\end{abstract}

Keywords : Behavioral experiment, verbal protocol, daily life, retrospective interview, subject selection, car navigation system, baseball game watching

\section{Introduction}

The research outcomes of science and technology are ultimately used by people in the form of products, systems, or services. Of course, there are R\&Ds for some elements of a system as well as R\&Ds for the materials used as parts, but eventually all of them will be used by people. Therefore, as synthesiology for utilizing the results of science and technology in society, it is necessary to have a research methodology to design the product or service that people perceive as being beneficial in their daily lives.

Ever since the Industrial Revolution, various industrial products entered the people's lives through mass production technology. However, as the living standards increased, people are no longer satisfied with the current mass produced products, and there are demands for products custom fit for the individual. It is the age of different strokes for different people. The traditional mass production is limited in responding to the people's diversified tastes, and the research is done for minimal manufacturing ${ }^{[1]}$ and ondemand manufacturing technology ${ }^{[2]}$ that can respond to low-volume high-mix production and built-to-order (BTO). In the service industry, it is recognized that a person may want something different according to the time and occasion, and the age of different strokes for a single person has arrived $\mathrm{d}^{[3]}$. For example, in the information service, providing information that pleases the user at a certain moment is expected to be realized based on the location information through GPS and optical communication ${ }^{[4]}$. The industrial technology is expected to develop the products and services that can fulfill various demands for various people, but the methodology for providing the policy for realizing what function and which service has not been established.

If the product or service is already accepted in society, the improvement of the function of that technology will be the objective of the $R \& D$, and the researcher can set the $R \& D$ scenario by applying the knowledge of natural sciences involved. However, this will not generate a novel product. When conducting the technological development of a novel product, the developer comes up with an idea, the idea is reviewed, and the research is conducted under the decision that it may be accepted by the users. However, that does not necessarily result in a hit product. This is not only because the individuality of the developer and the user is different, but it is also because it is impossible to switch the viewpoint completely to the user's side when one is on the developer's side. The reason why it is difficult to imagine the actual usage situations from the developer's viewpoint is because the product and service are used in various situations, including the user him/herself, that changes in various ways. Therefore, there must be a process early in the stage of R\&D to understand the actual usage situations of the person with certain personality traits, and obtain hints needed for designing the product and service for such a person.

1. Human Technology Research Institute, AIST Tsukuba Central 6, 1-1-1 Higashi, Tsukuba 305-8566, Japan * E-mail : akamatsu-m@aist.go.jp, 2. Center for Service Research, AIST Tsukuba Central 2, 1-1-1 Umezono, Tsukuba 305-8568, Japan

Original manuscript received February 14, 2011, Revisions received May 31, 2011, Accepted June 1, 2011 


\section{Why cognitive behavior in actual daily scenes?}

In ergonomics, a discipline to realize the product and system compatible with humans, the first thing to do is to correctly understand the characteristics of human users. Therefore, the human characteristics are studied by using the knowledge and methodology of psychology, physiology, medicine, and engineering, such as mechanical and information engineering. These methods attempt to understand humans in the framework of natural sciences. In the natural science approach, factors that are irrelevant to the essence of the phenomena under investigation are considered as disturbances, and it is very important to control such disturbances. If the researcher sets up a laboratory experiment that reconstructs the environment in which the product is used by appropriately controlling the disturbances, it is possible to create the product and environment that is compatible with the sensory function of, for example, elderly people ${ }^{[5]}$. This approach is effective in cases where the research subject is the interface between the person and the product or system, such as the design for display or shape of the product. However, there is a limit to studying the function and contents of the product, system, or service. That is because the user perceives the value by using the product or system or receiving the service in the context of daily life, and not in the laboratory ${ }^{\text {Note } 1)}$.

As a person lives in the daily life, the environment constantly changes and the individual recieves various stimuli and information. The brain and the body change to adapt to them, and behavior occurs. People interact continuously with the ever-changing environment, and the individual also changes

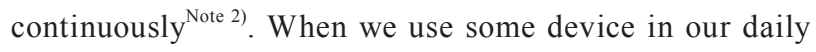
life, we behave not only according to the device, but also according to the situation in which the device is placed. For example, when one is using a copy machine, one's behavior is affected by the way the paper is being discharged, the placement of the lid of the device, and the operations one had been doing, and not just according to what is displayed on the copy machine display. This is called the situated action ${ }^{[6]}$. Therefore, unless the person is using the product or receiving the service in the actual situation, it is difficult to correctly know how the person uses the product or receives the service.

What is frequently done to obtain the evaluation of usage is to make a prototype, have some assumed user try out the product in a trade show or an exhibition, obtain the comments on it, and find points that must be improved ${ }^{[4][7]}$. However, against our intentions, it is difficult to reproduce the usage in actual daily life with this approach. Also, since the starting point is the realization of the requirement imagined by the developer side, the true requirement of the user may not be met even if improvements are made. The developer must start by understanding the user's situation of use and then make a product that is highly likely to satisfy the user. Therefore, a research method that clarifies what is needed by the person, what the person wants, and what pleases the person is necessary, to discern the user's true requirement that may not have been imagined by the developer. This method is not a pure cognitive science method to merely understand the cognitive behavior of the person, but the content obtained from the research must lead to designing the functions of the product or service.

Most of the human behavior is carried out unconsciously, and a research method that allows analysis of how the person felt and how he/she made decisions done on-site is necessary, rather than a post-event method like a questionnaire survey. In this paper, we explain the cognitive chrono-ethnography (CCE) that was developed to understand humans by incorporating the chronological changes of the humans and environment based on the cognitive science method. The research scenario for this method is comprised of the following processes: first, the critical parameters that lead to the design of the product or service are reviewed to construct a hypothesis; the experiment is conducted in actual daily conditions that is controlled based on the critical parameters; then the adequacy of the hypothesis on the human behavior is verified from the analysis; and the function that should be realized in the product or service is proposed.

\section{Development of the cognitive chrono- ethnography method}

\subsection{Retrospective interview}

In the 1970s, the verbal protocol method was proposed for analyzing the cognitive process in the field of cognitive science ${ }^{[8]}$. This is a method where a person is asked to verbalize and express whatever comes to mind on site. As mentioned earlier, humans select their actions continuously based on the limited cues obtained in certain situations, even if the selection is not necessarily optimal for achieving the goal. However, when explaining one's actions to another after the event, one gives explanations as if there is total rationality in the series of actions that are remembered in fragments, to make one's actions meaningful. Therefore, the verbal expression of past events is considered to be unreliable. In the case of the verbal protocol method, the advantage is that the subject cannot reconstruct and offer rationalizing explanation since the verbalization is done in real time. This method is used often in the usability testing of information device, but the subject may be too focused on verbalizing whatever comes to mind at each action and this may interfere with the operation of the device. Although this may not be a problem when the device is operated in the laboratory, it is not readily applicable when studying the cognitive behavior in daily life.

In the case where the verbalization of the cognitive content cannot be done in real time, the only method is to look back afterwards, but this cannot be done by relying solely on 
the subject's memory. Therefore, we proposed a method of recording the video images of the environment in which the behavior took place and the behavior itself, and then have the subject report what triggered the action while viewing the video replay ${ }^{[9]}$. In the study of learning and memory in psychology, it is known that the memory of the event at the time (episode memory) can be recalled when the subject visits the place or sees the object that was there. This is called the context-dependent memory ${ }^{[10]}$. Showing the image from the subject's viewpoint that changes from moment to moment along with the behavior provides hints for recalling the environmental situation. It is considered useful for reporting the cognition and decision recalled by the subject in the situation.

In the case where the subject is asked to verbalize the cognitive process at the time as the past behavior is reexperienced through recorded images, the method can be considered as the retrospective verbal protocol method. On the other hand, in the case where the subject is asked to describe the cognition and decision processes of only the behavior that the experimenter wishes to focus upon, the method is an interview, and this is called the retrospective interview method. Since it is not necessary to verbalize all of the daily actions if the aim is for designing the product or service, a retrospective interview method will be used where the interviewer picks up the action that may be related to designing and asks question ${ }^{\text {Note } 3)}$.

\subsection{Chrono-ethnography}

Ethnography is the sociological methodology to understand the people's activities in society ${ }^{[11]}$. The word means a research method to describe (graph) the people (ethno) by entering the social group to observe the behavior of the constituents of society, conducting interviews to the people, and then describing the activities of the people in that society through language ${ }^{\text {Note 4) }}$. In the sense that the method involves recording and analysis of the people's activities by focusing on the cognitive behavior in real life situations, it can be said that the retrospective verbal protocol and retrospective interview methods are the combination of cognitive science and ethnographical methods ${ }^{\text {Note 5) }}$. While the original objective of ethnography is to understand the social activities as they are, the combined method that controls the experimental conditions as explained in the following chapter is effective for designing the product or service. This is because it is difficult to obtain findings that can be utilized for product or service design from the research that attempts to merely understand and recognize human beings.

Since whether the person perceives the product or service as pleasing depends on the chronological history such as what cognitive features the person has, what history, and under what circumstances this person grew up, we decided to treat the chronological history explicity. Therefore, since the emphasis will be on the person who changes over time and the detailed description of the cognitive process of the person placed under a situation, we call this method the cognitive chrono-ethnography (CCE) by adding chrono- (for time) and cognitive to ethnography.

\subsection{Elite monitor and critical parameters}

Through joint researches with companies that wanted to investigate the human cognitive behavior for constructing a product or service, we have developed the cognitive chrono-ethnography method, as we applied the retrospective interview method as a practical method using the recorded images of the behavior scenes using a small camera attached to the head. This was used for studying the daily scenes such as the behavior of elderly people in train stations or driving automobiles. While the details cannot be described fully due to the limitation of space of the paper, it was found that the behavioral characteristics for individuals could be clarified by selecting certain types of subjects based on preliminary hypothesis, and using them as the participants of the experiment. In the case of the behavior of elderly people in train stations, we set the hypothesis that there were differences in the cognitive functions of the elderly and these cause differences in the behavior. We hypothesized that there were people with impaired attentive functions, working memory functions, and planning functions. The hypothesis was investigated by creating an experimental procedure that may reveal these differences ${ }^{[12]}$. Starting with the hypothesis, the method for selecting a typical person based on the hypothesis is considered (in the above example, the AIST cognitive function test was developed), the actions of the different types of selected people are compared, and the difference in the behavioral characteristics of individuals is clarified to verify the hypothesis. The experimental participant with typical characteristics is called the elite monitor. By using the elite monitor, it is possible to obtain hints for the product or service design for a certain characteristic $^{\text {Note 6) }}$. By integrating the abductive research method for hypothesizing the difference in characteristics and the retrospective interview method, we constructed the cognitive chrono-ethnography method that will provide useful findings for the product or service design.

While the ethnography research places importance on describing everything as they are, in the approach of synthesiological method for designing the product or service, it is important to seek the critical parameters that can be used for design. The critical parameters include, for example, the parameter to determine whether the experiment participant is a target user, or the timing of information presentation that gives the impression that the provided information is useful. Therefore, if the candidates for the critical parameters are found through preliminary observation study, the experiment using the actual scenes is conducted by controlling and recording the critical parameters. When the elite monitors 
with typical target user characteristics or the person with typical characteristics for the subjects under investigation (for example, skilled service provider) are selected and used as the experiment participant, it is possible to clarify the product or service that may please that person.

\subsection{Research process}

Through the research projects described above, the CCE research method was established. However, as a research method, it must be used widely rather than be used by only certain researchers. Therefore, research experiences were reviewed, and the whole process was clarified so it could be used as a universal research method. Since there were some parts where the optimal method was not taken when we reviewed individual research projects, the research process was established incorporating the revisions.

The main process is shown as follows. Before the experimental investigation process, the hypothesis is formed by observing the relationship between people and an object (or among people) in the daily situation under investigation, and the elite monitor is selected by some appropriate method. The participants are asked to behave under control of the critical parameters in a daily situation, and the video image from the point of view of the participant is recorded along with other related events including the uncontrolled parameters. A retrospective interview is done for the recorded actions and the cognitive behavior process is described. After the investigation, the findings for the product or service design are extracted and the hypothesis is verified.

The details of the process are described in references [13] and [14]. The CCE can be organized into the following six steps to be followed by the researcher:

(1) The basic survey method such as ethnography and observation study is used to see the rough structure of the action selection process of a person in the situation under investigation.

(2) Based on the behavioral and cognitive characteristics of the person that have been clarified under current knowledge, what kind of characteristics, knowledge, or environmental factors are involved in the action selection of a person is considered based on the result in (1). The critical parameters are identified and the hypothesis for the cause-effect structure is formed.

(3) Based on this hypothesis, the person with typical behavior characteristics is selected among various people that comprise the group under investigation, and the selection criteria and recruiting method for the elite monitors are created.

(4) The elite monitors are selected. They are asked to behave in the situation under investigation, and their behaviors are recorded.

(5) Using the recorded behavior, the action selection process is analyzed, compared with the hypothesis established in (2), and the adequacy of the hypothesis is discussed.

(6) If the result is unsatisfying, the investigation is repeated by reconsidering the method by returning to (2). If it produces satisfying results, the findings that may aid the product or service design are organized from the results.

This was the process of development of the cognitive chrono-ethnography research method and its concepts. We present specific examples in chapter 4 .

\section{Application of CCE}

\subsection{Providing of smart information in driving}

To realize a car navigation that provides useful information to assist driving, we conducted a joint research with an automobile manufacturer using the CCE. While useful information can be defined as the information that pleases the driver, we started by investigating whether there is information that people actually find pleasing. People may think that a human passenger will be capable of providing pleasing information compared to a car navigation that is a machine, but that is not always true. Therefore, the creation of a passenger who can provide information that the driver finds pleasing was conducted as part of the research process. Since the same information may or may not please the driver, the requirement was that the passenger knew the driver well. Therefore, it was not just a driver and a passenger, but the relationship between the driver and the passenger who could provide smart information was identified as the critical parameter to determine the elite monitor.

To obtain the passenger who knew the driver well, pairs such as married couples and friends who went driving together frequently were gathered through an Internet questionnaire. Among the 1,600 people gathered, 10 driver-passenger pairs were selected as elite monitors based on the questions on whether the partner was "officious", "attentive", "considerate", and others. Interviews were done to confirm both of them were considerate. The destination that one of the pair knew but the other didn't was selected, and the advice points for the route were described by the one who knew. The results were carefully examined, and four pairs who were expected to provide much thoughtful information were selected as the elite monitors of the experiment.

Even if the monitors were selected through this process, it was uncertain that the information that the person considered thoughtful was truly pleasing and useful for the driver, and it might be that the person simply believed so. To solve this problem, three experimental driving trips were conducted. In the first driving trip, the information was provided according to the information that the passenger thought would be 
thoughtful. After the drive, the pair was shown the video image of the conversation recorded along with the road scenes (Fig. 1), and the driver was asked to evaluate whether the provided information was thoughtful. Also, what points were useful and if not where lay the problem were extracted through the retrospective interview. The passenger learned what the driver considered useful, and the second driving trip was conducted. The same process was done after the second trip. The findings obtained from other pairs were shared to see the points that were perceived as being thoughtful, and the third driving trip was done. The driver and the passenger changed places and the same procedure was applied. They could learn what was the thoughtful information from both standpoints. By using the elite monitor who knew the route, knew each other well, and knew what information pleased the driver, we were able to clarify the information provision for somewhat ideal driving assistance ${ }^{[15]}$.

The dialog contents extracted in this process were categorized into ones for road guidance such as need of right or left turns, safe and smooth driving, information about the facilities and sightseeing points to enjoy the area, and others. For each information provided, the content, the provision method of timing and ways of instruction, the situation of the road such as the width, and whether the driver's workload was high or not were described (Table 1). These were the critical parameters since they influenced whether the provided information was perceived as thoughtful or not. Of the extracted information, the information that was perceived as thoughtful was categorized into those that corrected the driving action, those that assisted or enhanced the driving action, and others (Table 2). For example, the correction of driving action is information provided when the passenger thinks that a certain driving action is necessary for the driver in the current situation (for example, early lane change or something that will make driving easier later), but the driver may not take that action. If the information
Table 1. Attributes to describe the provided information

\begin{tabular}{|l|l|l|}
\hline \multicolumn{1}{|c|}{ Attribute } & \multicolumn{1}{|c|}{ Explanation } & \multicolumn{1}{c|}{ Value } \\
\hline Provided content & $\begin{array}{l}\text { Information provided } \\
\text { by verbalization }\end{array}$ & Specific content \\
\hline Provision method & $\begin{array}{l}\text { Information communication } \\
\text { method }\end{array}$ & Timing, instruction method \\
\hline Road situation & $\begin{array}{l}\text { Road situation during } \\
\text { verbalization }\end{array}$ & Road width, speed, etc. \\
\hline Driver's condition & $\begin{array}{l}\text { Driver's condition during } \\
\text { verbalization }\end{array}$ & $\begin{array}{l}\text { Driving workload, } \\
\text { assumed knowledge }\end{array}$ \\
\hline
\end{tabular}

Table 2. Motivation for information provision by the passenger

\begin{tabular}{|l|l|}
\hline $\begin{array}{l}\text { Correction } \\
\text { of driving action }\end{array}$ & $\begin{array}{l}\text { The passenger thinks that a certain driving action is } \\
\text { necessary for the driver to drive in the current situation, } \\
\text { but thinks that the driver is not likely to take the action. }\end{array}$ \\
\hline $\begin{array}{l}\text { Assistance } \\
\text { of driving action }\end{array}$ & $\begin{array}{l}\text { The passenger thinks that a certain driving action is } \\
\text { necessary for the driver to drive in the current situation, } \\
\text { and thinks that the driver is likely to take the action, } \\
\text { and the driving action will be encouraged if the information } \\
\text { is given. }\end{array}$ \\
\hline $\begin{array}{l}\text { Addition } \\
\text { of supplementary } \\
\text { information }\end{array}$ & $\begin{array}{l}\text { The passenger thinks that the driving satisfaction will } \\
\text { increase if the driver is given some information in the } \\
\text { current situation. }\end{array}$ \\
\hline
\end{tabular}

is provided at an appropriate timing such as after passing through the intersection and the traffic flow becomes smooth, it is perceived to be thoughtful advice. Through this study we were able to clarify the structure of the provision of thoughtful information.

\subsection{Growth stage of a baseball fan}

The method to study the thoughtful information provision in driving involves setting the scenario of taking a trip to an unknown destination and training (or nurturing) the elite monitor. This is called the scenario-oriented CCE. In contrast, the approach involving the observation of people in their daily situations without a scenario is called the non-scenario oriented CCE. As an example, we describe the application to the study of spectator behavior at a baseball game.

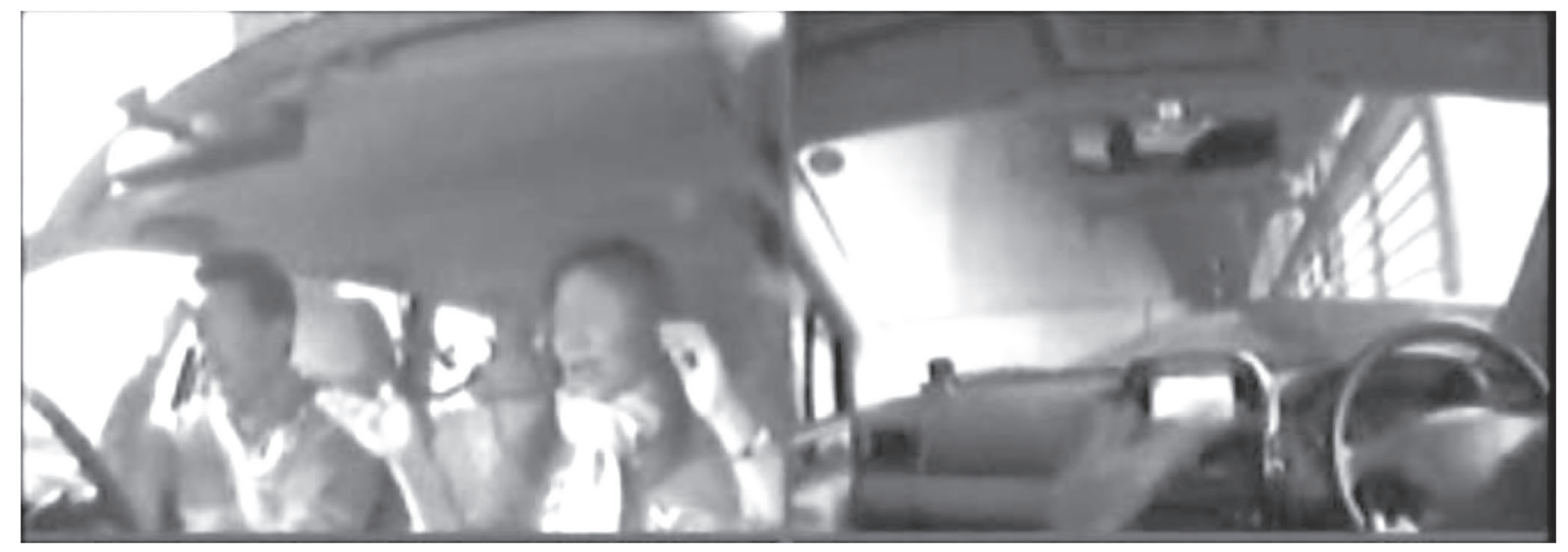

Fig. 1 Monitors during the driving trip 
In the service that provides sports entertainment, it is important first to get people to the ballpark or the soccer stadium, and the next issue is what kind of experience can be given there. People who come to watch sports include those who come to the stadium for the first time and those who attend frequently or the repeaters. Even a repeater had the first time when he/she attended the game. Before that, the person most likely watched sports on television, and turned into a repeated game goer through various information and experiences. The objective of the research was to clarify the structure of the shift or how a sports watcher turned into a repeater.

We investigated the critical parameters that determined how the repeater of baseball game watching enjoyed the baseball game. First was the objective and enjoyment of going to the stadium, second was the degree of information exchange about the baseball team with the surrounding people, third was the strong feeling of support as a team fan at the games, and fourth was the personality (behavioral characteristics) of the person. From this perspective, 30 elite monitor candidates were selected based on the response to the Internet questionnaire survey of about 1,000 members of the baseball team fan club. Five males and five females were selected from the three enjoyment types including "sports watching is my personal enjoyment", "it is enjoyment for the entire family", and "it is enjoyable but I don't really want to become a hardcore fan". These three types were found from the cluster analysis of the questionnaire survey. Each group contained differing critical parameters. Group interview was held with 30 people, and they were mapped on the twodimensional graph composed of the axis for how they enjoy the game (from people who enjoy the game itself to those who enjoy cheering for the team) and the axis of fan maturity (from hardcore who goes to fan service events as well as the games, to those who love baseball but have no time to watch the games). Then, nine people were selected as elite monitors, making sure there were no biases. The elite monitors were asked to watch the games at the stadium, and the behavior of the monitors, the progress of the game, and the atmosphere of the spectators' stands were recorded on the video. Heart rate of the monitor was also recorded as the physiological measure to see the state of physical excitement during the game. Retrospective interview was done, and the feelings they had during the games were extracted as the monitor watched the video (Fig. 2). Also, other sports watching behavior the elite monitors engaged in the past were surveyed through the interview.

From this survey, the stages of the fan were categorized into the following three: pre-fan who "knows/doesn't know baseball but is not interested in professional baseball games", fan who "has become fond of the team and goes to watch the game at the stadium", and the repeater who "goes to the games enthusiastically, has his/her own watching style, and consciously makes time to go to the games" (Fig. 3). The motives that were given for shifting from pre-fan to fan included the introduction of a star player, the expectation for league victory, and the amazement at the cheering fans at the game that one happened to attend. The motives for shifting from fan to repeater were the sense of unity with the other spectators at the stadium that does not get communicated through $\mathrm{TV}$, the accumulation of detailed knowledge about the players, and the aquisition of companions such as family or friends with whom one can watch the games together. There were several paths for the shifts, and there were cases whereby "the person who did not know anything

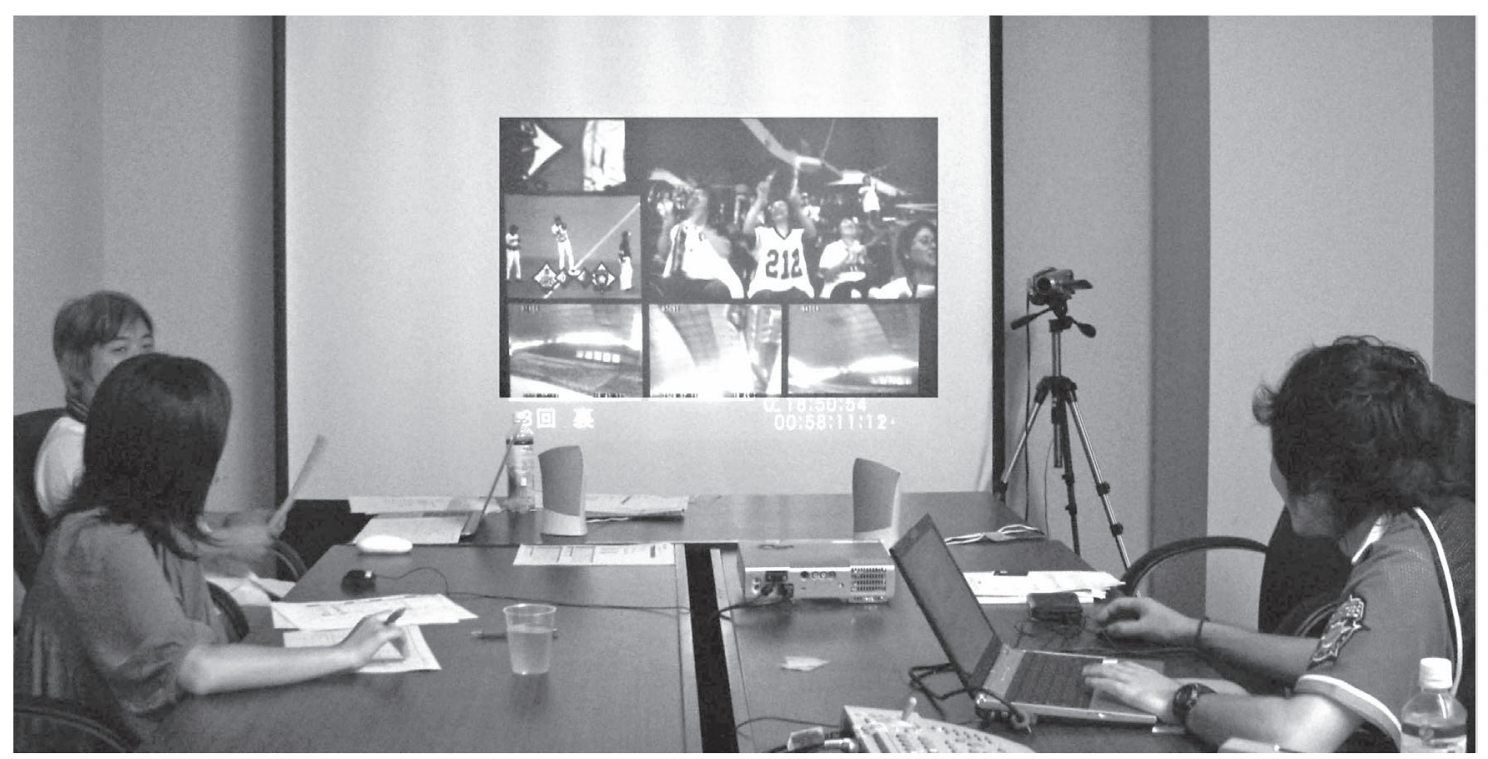

Fig. 2 Interview to baseball fans after game watching at the stadium 
about baseball" became a fan because he/she was amazed by the excitement at the stadium, voluntarily participated in cheering, and became adherent to the total experience of cheering and the game. Another case was that "the person who knew baseball and was interested in professional baseball" became emotionally involved in the players and the team through the real game, and became a person who enjoyed the game, or enjoyed both the game and cheering (Fig. 4). By using the CCE, we were able to clarify the structure of fan growth, or how a person who was not really interested in baseball became a repeater and how they enjoyed the game.

\section{Application to product or service design}

After obtaining the clues for identifying the function and service that the target user wants by the CCE method, we move into the $R \& D$ process for concretizing the product or service. For example, if we know that well-timed, useful information from a considerate passenger is given when and about what,

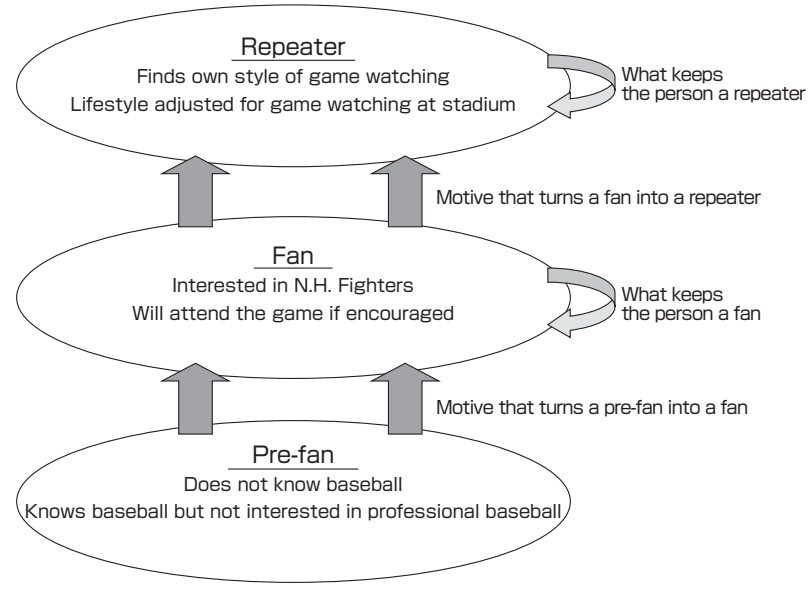

we can set the development goal of providing such information from the car navigation system. Since good timing by which the information is provided is the critical parameter of whether the person perceives the information as being thoughtful, the technological issue will be whether the system can determine the timing. Therefore, we conducted an experiment where the content and the timing decision for providing the information were defined as clearly written rules, and the information provision was done according to the rule. Although the information provision is done by a human operator, rather than changing the information provision flexibly, a manual is followed to simulate the information provision by a system using this rule. Moreover, to investigate whether it is possible to determine the timing according to the traffic condition and the driver condition without the passenger, we investigate whether an information provider (an operator) on a separate vehicle can provide the information at appropriate timing based on the image and sound information from the cameras in the subject vehicle via radio communication. This research is still in progress, but by providing the information via radio communication from a separate vehicle, the cues for timing decision is becoming clear, and the system may be able to determine the timing using sensors ${ }^{[16]}$. In case of information provision by a device, the function of the product will become clear through these research processes, and the function to be realized will become concretized.

In the case of service, if, for example, the motive for a baseball fan to rise from an "occasional game watcher" to a "repeater" becomes clear, the baseball team owners can plan the actions to be taken. They can determine how they can reach out to the target subjects, how they can provide the motives, and how to set the incentives. Of course, several, and not just one plan, can be considered.

Fig. 3 Three stages of fan maturity

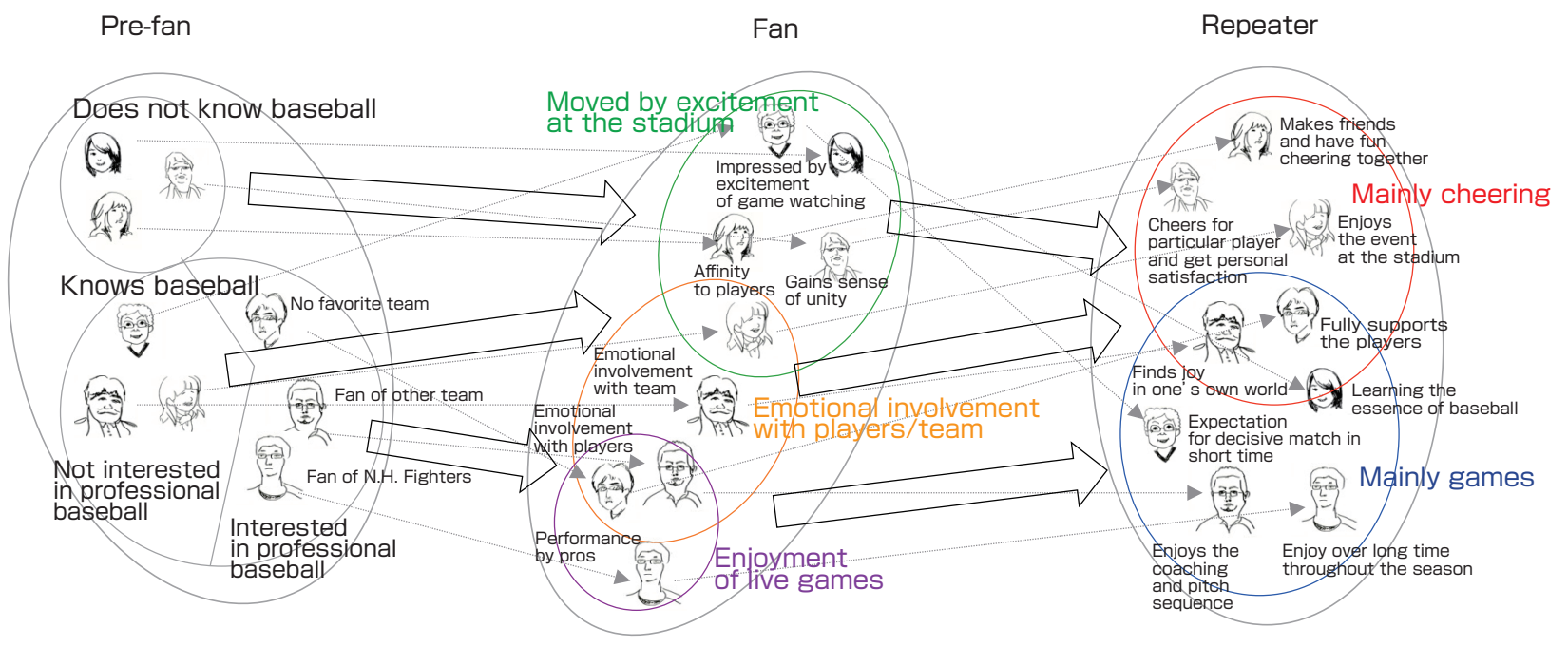

Fig. 4 Path of fan stage 
Whether they are products or service, the design is constructed based on the findings obtained by the CCE method, and the specific requirements of the plan and the prototypes with functions to be realized are created. The ways to realize them are reviewed, and realistic specifications are created, considering the current technology level and the service provision environment. In some cases, the decision may be that the product cannot be realized with the current technology level, and the plan must be terminated. The prototype is built based on the specification, the trials are done using the expected target user under the target situation, and the evaluation is obtained. In case of a product, since development cost and time are needed, it is necessary to conduct the experimental evaluations at various stages of the development process to make sure the project does not wander in the wrong direction. However, in case of service, the service can be implemented more easily compared to products, and the new service can be incorporated into the actual service provision process. The survey is done by executing the service, seeing that the service expected by the service designer is provided to the target user, observing whether the service is effective, and improving the plan by understanding where the problem lies if the service does not seem effective. By turning this cycle of the hypothesis testing, the service provided is made compatible with the user ${ }^{[17]}$.

\section{Discussion}

From the analysis of the Synthesiology papers, Kobayashi et al. indicate that the way of synthesis when realizing the product to be used in society by integrating the elemental technologies differs according to the technological field ${ }^{[18]}$. For example, in the research fields that handle the materials themselves such as nanotechnology, and device technology, the researcher can utilize the knowledge of natural sciences, clarify the elemental technologies and the factors that affect the subjects, and select what must be done for synthesis. On the other hand, in the research fields that handle daily living and in some life sciences, the researcher may take the spiral approach where the product or service is introduced to society and advances are made as evaluations are obtained, because not only are there multiple factors, but also because there are many unknown factors. However, unless the product or service that is being introduced initially has high prospect for acceptance in society, it will not diffuse in society even in the spiral approach. To prevent such failure, it is necessary to have a method that allows the factors and elemental technologies for the acceptance of the product or service to be clarified as much as possible and to be examined beforehand. The CCE method extracts and structurizes the variables for the target user in the actual usage situations, and aims to take a similar approach as the synthetic research in the field of material science, in the sense that the factors that affect the cognitive behavior under investigation are clarified and what must be selected for synthesis is determined.
Although it is similar to the research approach for materials, it does not have the same level of preciseness of natural science. Although hypothetical field experiments are done for the selection of the elite monitor and the critical parameter, it is difficult to show the validity of the hypothesis through statistical testing of the results. In CCE, there are only a few participants of the experiment as elite monitors. There may be only a few chances once or twice a day to make a certain situation constant when conducting the field experiment. For example, if the research involves railway use, the time zone when the experiment can be conducted is limited if the researcher wishes to avoid the effect of crowding. Also, there is no exactly-the-same action taken in the actual daily scene, and the analysis may remain qualitative. For example, if the majority of a certain category of experimental participants takes the same behavior and the behavior is not seen in other categories, it can be concluded that the categorization hypothesis was not wrong. Therefore, it is not hypothesis testing in the natural scientific sense. The natural scientific hypothesis testing may be possible if the scale of experiment is expanded, but considering that the same situation is not repeated in actual daily situations, the testing by reproducibility would be essentially impossible. The everyday situation in which we use the product or service changes continuously and we are never subject to the same situation. Therefore, we must be aware that there is a limit on depending on the natural scientific methodology. However, if we abandon the natural scientific research approach for understanding the factors that affect the phenomenon, we cannot reduce the gap to obtain acceptance of the product or service in society.

In the sense that research subjects are daily situations, the $\mathrm{CCE}$ is similar to the sociological method. Ethnography as a sociological method aims to understand society, or to seek the behavioral principles common to human groups or society by describing the behavior of humans in society just as they are $^{[11] \text { Note } 7)}$. In contrast, the CCE clarifies the differences in individual characteristics and adds experimental control such as setting the situation. This is a synthetic research approach for the purpose of constructing the product or service, and the insight for designing the product or service can be obtained by introducing the elite monitor and the critical parameter. If the product or service is constructed based on the findings of $\mathrm{CCE}$, and the product or service becomes more acceptable to the user by turning the cycle of evaluation, then the validity of the findings obtained can be investigated, and the product or service acceptable to society can be realized.

It is not easy to realize a good product or service by thinking inside the developer's mind only. By using CCE, the product or service can be designed effectively. In the evaluation for the usage, the cycle of improvement and optimization can work efficiently by clarifying the viewpoints of function of the product or service. 


\section{Notes}

Note 1) Experimental psychology to investigate the human sensory functions by controlling the stimuli in a laboratory setting was started by Wilhelm Wundt of the Universität Leipzig in 1879. However, Wundt indicated the necessity of psychology in the actual evironment, since it was impossible to discern the higher-level cognitive functions such as thought and problem solving by laboratory research.

Note 2) MHP/RT is the computational model for the action selection process taking in consideration this brain mechanism. It provides the logical framework of the CCE method, but the explanation was omitted here due to space limitation ${ }^{[13]}$.

Note 3) The retrospective interview is conducted to have the subject report the contents of memory that was activated during the action selection. The memory that was activated during the action selection is encoded and stored in conjunction to the situation or the context. It is known that the reproducibility is good if the context when the memory is recalled is similar to the context when it is memorized (context dependency of memory). It is also known that the subject's report is affected by the way the interviewer asks the questions. There is a phenomenon called the "post-event information effect" in which the subject tends to report the information given after the event or the mixture of the original and post-event information if the subject is given information related to the event after he/she experiences the event. The action selection of the subject is done under bounded rationality (where the decision is based on limited views and limited cues, rather than making a rational decision that directs the goal and the totality of the situation) that is a human characteristic of decision-making. The retrospective interview, which is an important process of CCE, is designed to extract the cognitive behavior process according to bounded rationality, without the influence of post-event effect.

Note 4) The efficacy of the sociological method as a method of technology transfer, whereby the problem is solved by entering the organization under investigation is addressed by Kinoshita et al. ${ }^{[19]}$

Note 5) An example of the famous ethnographical research in the field of cognitive science is the study of cognitive process of the crew in a cockpit of an aircraft, conducted by E. Hutchins et al. Here, the researchers entered the cockpit to observe the behavior of the crew and then conducted interviews.

Note 6) The person who is expected to use a certain product is called the target user. The persona method is widely used for product development assessing highly individualistic target users. However, this method is used to clarify the target user in the development process, and the evaluation for how the expected user actually uses the product or service remains within the realm of the developer's imagination.
Note 7) Among the ethnographic research methods in sociology, the action research method aims to find the problem in society or organization and to seek the solution to that problem.

\section{References}

[1] J. Akedo, S. Nakano, J.H. Park, S. Baba and K. Ashida: The aerosol deposition method - For production of high performance microdevices with low cost and low energy consumption, Synthesiology, 1 (2), 130-138 (2008)(in Japanese) (Synthesiology English edition, 1 (2), 121-130 (2008)).

[2] T. Kamata, M. Yoshida, T. Kodzasa, S. Uemura, S. Hoshino and N. Takada: Development of flexible-printable device processing technology - For achievement of prosumer electronics, Synthesiology, 1 (3), 190-200 (2008) (in Japanese) (Synthesiology English edition, 1 (3), 177-186 (2009)).

[3] M. Usui: Advancement of the service industry and the value co-creating society, Zukai Sebun-Irebun Ryu Sabisu Inobeshon No Joken (Illustrated Conditions for SevenEleven Style Service Innovation), 160-170, Nikkei BP (2009) (in Japanese).

[4] H. Nakashima and K. Hasida: Cyber Assist project as service science - A project that began ten years too early, Synthesiology, 3 (2), 96-111 (2010) (in Japanese) (Synthesiology English edition, 3 (2), 107-123 (2010)).

[5] K. Kurakata and K. Sagawa: Development and standardization of accessible design technologies that address the needs of senior citizens - Product design methodology based on measurements of domestic sounds and hearing characteristics, Synthesiology, 1 (1), 15-23 (2008) (in Japanese) (Synthesiology English edition, 1 (1), 15-23 (2008)).

[6] Lucy A. Suchman: Plans and Situated Actions: The Problems of Human and Machine Communications, Cambridge University Press (1987) [Y. Saeki et al. trans.: Puran To Jokyoteki Koi: Ningen-Kikai Komyunikeshon No Kanosei, Sangyo Tosho (1999) (in Japanese)].

[7] Y. Satoh and K. Sakaue: A secure and reliable next generation mobility - An intelligent electric wheelchair with a stereo omni-directional camera system, Synthesiology, 2 (2), 113-126 (2009) (in Japanese) (Synthesiology English edition, 2 (2), 107-120 (2009)).

[8] Y. Kinoe: Measurement of thought process by verbal protocol, Ningen Keisoku Handobukku (Handbook of Human Measurement), Asakura Publishing (2003) (in Japanese).

[9] M. Akamatsu, R. Kasahara and M. Obata: Driving task analysis by verbal report using recorded images of driving behavior, J. Human Interface Society, 4 (2), 93-102 (2002) (in Japanese).

[10] S. M. Smith and E. Vela: Environmental context dependent memory: A review and meta-analysis, Psychonomic Bulletin \& Review, 8, 203-220 (2001).

[11] U. Flick: Qualitative Forschung: Ein Handbuch, Rowohlt TB-V (2002) [H. Oda, N. Yamamoto, J. Kasuga and N. Miyaji trans.: Shitsuteki Kenkyu Nyumon, Shunjusha Publishing (2002) (in Japanese)].

[12] M. Kitajima, T. Kumada, H. Ogi, M. Akamatsu, H. Tahira and $\mathrm{H}$. Yamazaki: Usability of guide signs at railway stations for elderly passengers - Focusing on planning, attention and 
working memory, The Japanese Journal of Ergonomics, 44 (3), 131-143 (2008) (in Japanese).

[13] M. Kitajima and K. Naito eds.: Shohisha Kodo No Kagaku: Sabisu Kogaku No Tame No Riron To Jissen (Science of Consumer Behavior: Theories and Practices for Service Engineering), Tokyo Denki University Publishing (2010) (in Japanese).

[14] M. Kitajima and M. Toyoda: CCE (Cognitive ChronoEthnography) No Jissenteki Gaisetsu: Ninchi Kagaku Ni Motozuku Hito No Kodo Seitai No Chosa Shuho (Practical Overview of CCE: Survey Method for Human Behavioral Ecology Based on Cognitive Science), On Book (2011) (in Japanese).

[15] Y. Maruyama, K. Kuroda, K. Katou, S. Kitazaki, Y. Minowa, K. Inagaki, T. Kajikawa, M. Kitajima and M. Akamatsu: A study of useful information for driver assistance, JSEA Transaction, 40 (2), 537-543 (2009) (in Japanese).

[16] Y. Minowa, K. Inagaki, T. Kajikawa, K. Kuroda, K. Omori, T. Kitazaki, M. Kitajima and M. Akamatsu: Review of the experiment by simulated navigation system to check the efficacy of "information useful to the driver", Proceedings of Society of Automotive Engineers of Japan, Autumn 2010, 99-10 (2010) (in Japanese).

[17] K. Naito ed.: Sabisu Kogaku Nyumon (Introduction to Service Engineering), The University of Tokyo Press (2009) (in Japanese).

[18] Synthesiology Workshop: Methodology of technology integration toward establishing an open innovation hub, Synthesiology, 4 (1), $52-58$ (2011) (in Japanese) (Synthesiology English edition, 4 (1), 63-69 (2011)).

[19] Y. Kinoshita and T. Takai: A field-scientific approach to Clinico-Informatics - Towards a methodology for technology transfers, Synthesiology, 3 (1), 36-46 (2010) (in Japanese) (Synthesiology English edition, 3 (1), $64-76$ (2010)).

\section{Authors}

\section{Motoyuki AKamatsu}

Completed the doctorate course at the Department of Administration Engineering, Graduate School of Engineering, Keio University in 1983. Doctor of Engineering. Joined the Industrial Product Research Institute, Agency of Industrial Science and Technology in April 1986. Engaged in the researches for tactile perception, brain mechanism for sensory-motor integration, computer input device, ITS human interface, and car driving behavior. Currently, director of the Human Technology Research Institute, AIST. Professor of the Cooperative Graduate School, Tsukuba University. In this paper, reviewed the comparison and relevance to other researches to position this research in the field of synthesiology, and constructed the whole structure of the paper.

\section{Muneo KitaJima}

Completed the master's course at the Department of Physics, Graduate School of Engineering, Tokyo Institute of Technology in 1980. Doctor of Engineering (Waseda University, 1986). Joined the Industrial Product Research Institute, Agency of Industrial Science and Technology in April 1980. Engaged in the researches for reading device for vision-impaired people, cognitive

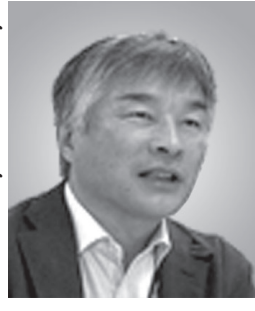
modeling in human-computer interaction, usability for hearingimpaired people, and action selection in everyday life. Currently, principal researcher, Center for Service Engineering, AIST. In this paper, was in charge of the development and application of the cognitive chrono-ethnography method.

\section{Discussions with Reviewers}

\section{Emphasis of the authors' thoughts and actions Comment (Akira Ono, AIST)}

This is an extremely interesting research, and it seems that the authors are creating a new discipline in synthesiology. On the other hand, I feel that the descriptions in this paper are rather explanatory and plain. I think the reader can better understand the significance of the research in terms of synthesiology if you emphasize your thoughts and actions, like what intentions the authors had, what they selected, and what they determined.

Answer (Motoyuki Akamatsu)

This paper not only recapitulates the research that we have been doing as synthesiology, but was written with an objective of positioning this research, while trying to define the overall picture of synthesiology. We tried to position our research by reviewing researches aiming to connect products to human users, and therefore, it has become rather explanatory. We revised some of the expressions to clarify our statements.

\section{Verification of the cognitive chrono-ethnography method Question (Akira Ono)}

There are two interesting case studies of the cognitive chrono-ethnography (CCE) method, and the results are steadily being accumulated. On the other hand, I understand that you have not yet gotten to the point where you have done verification by obtaining user evaluation after applying the results to the design of car navigation and baseball game programming. Am I correct? Also, how do you test and evaluate the research results of whether the past CCE research method was adequate or inadequate?

Question (Masaaki Mochimaru, Digital Human Research Center, AIST)

I found that the method of this research has analogy to the idea of process standard in the sense that the quantitative verification is not discussed. In process standard, the procedure and the method to check the procedure are standardized, and that whether the procedure is carried out properly can be confirmed. Moreover, a person other than the one who created the process can execute it. Analogically concern, can this CCE method be replicated by anyone if the procedure is followed?

\section{Answer (Motoyuki Akamatsu)}

We need more time for product development based on the result of this research. Thus, as you indicated, we have not reached the state of evaluation by actual users. From the standpoint of the verification being the acceptance of technology and service by society, I must say that it has not been verified yet. 
Before launching a product or service to society, a rationale will be needed for determining whether to give the go-ahead to the design of the product or service based on the findings of the CCE. Our current thinking is that the convincibility of the results obtained and the adequacy of the process are to be evaluated. The adequacy of the process is, for example, whether the procedure can extract the characteristics of target users of the product or service that one wishes to realize. However, we do not think that we will automatically reach the correct results as long as the process is carried out properly. Some sort of evaluation of the process or results is necessary. This evaluation may be difficult unless it is done by a person who has thorough understanding of the product or service under investigation, and it may be something like a peer review. In this type of research, I think it is important to do joint research with the people who have thorough understanding of the product or service, namely the people of the company that are providing the product or service and those who are capable of making evaluation.

\section{Reference to other Synthesiology papers Comment (Akira Ono)}

In this paper, the discussion is developed by citing the papers published in Synthesiology. The reviewer thinks that the synthesiology researches should be mutually contributing even though the contents are diverse. Please express, from the standpoint of the author who cited the references, how these affected this research.

\section{Answer (Motoyuki Akamatsu)}

The research described in this paper was started over 10 years ago, and I was aware that we were engaging in Type 2 Basic Research from the beginning. However, this research is an analytic research in the sense that it attempts to clarify the human behavioral characteristics, and I wasn't quite sure what points ought to be emphasized as a Synthesiology paper that focuses on synthetic research in which the elemental technologies are integrated and synthesized to realize some object to be used in society. I found a way during the workshop that I cited as reference [18] where I discussed the different approaches to synthesiology in different fields. I sought the characteristics of the different approaches by breaking them down into categories: the researches governed by principles of the nature as exemplified by physics; the engineering researches that assemble the elemental technologies; and the researches that involve people using the results in the form of products. I was able to see the similarities between our research and the material research approach where one understands what can be realized by pursuing the properties of materials. If the human cognitive behavior can be clarified through analysis, we can understand what kind of product or service can be realized. I think there is a common ground that knowing well will guide toward realizing a product. Our research is to have people use the product, but even in the same human technologies, we can now compare the engineering research that first focuses on manufacturing the product and the research that first focuses on understanding human behavior, like the ones we are doing. That allowed us to write a Synthesiology paper.

\section{Theoretical background of cognitive chrono- ethnography method \\ Comment (Masaaki Mochimaru)}

The characteristic of the CCE that is the core technology in this paper is organized well in relation to the other previous methods. Reading the cited references, I can understand the theoretical background that the CCE was created based on cognitive science. In this paper, I think you should briefly explain the theoretical background so the readers can understand just by reading this paper.

\section{Answer (Motoyuki Akamatsu)}

The background of developing the CCE was because of our awareness of the need to understand the action selection process after an event, using limited cues under certain situations. We tried to solve this problem by reproducing the condition when the action selection was done, and then by expressing the cognitive process using the working memory at that moment, to prevent describing the action as being selected based on total rationality, even though it was actually action selected from limited cues. The conventional method in coginitive science for not falling into explanation by total rationality is the verbal protocol method. Since retrospective interview and CCE are essentially based on that method, I added some description in the beginning of subchapter 3.1. For the researchers interested in the theoretical background, I added Note 4).

\section{Initial hypothesis Question1 (Masaaki Mochimaru)}

For the behavior observation, some degree of initial hypothesis (identifying the critical parameter and the selection of elite monitor by survey and observation) is needed, and the results obtained are applicable only in the set context and place based on the initial hypothesis. In that sense, isn't the framework of "findings that may be found" by the CCE is already determined at the point the initial hypothesis is set? Do you have the process of constructing the initial hypothesis properly as much as possible? Answer1 (Motoyuki Akamatsu)

As you indicated, the identification of the critical parameter and the selection of elite monitor are very important, and they will determine the framework of the conclusion obtained. However, the CCE is not a method for hypothesis testing research whose objective is to verify the initial hypothesis. That is because the hypothesis is tested to determine the framework of the product and service (car navigation or sports game attendance) such as the critical parameter and elite monitor, and it does not directly determine the framework of the contents or functions provided by the product or service. The process of CCE is designed to readily obtain the insights for designing the product or service, and by setting the initial hypothesis, I think it avoids doing research without a hypothesis and missing out the useful conclusion.

At any rate, although the initial hypothesis is extremely important, our thinking at this point is that when one constructs the initial hypothesis, one must look at the objective by eliminating any assumptions as much as possible while utilizing the researcher's perspicuity as much as possible. Speaking from our experiences, to construct a hypothesis by eliminating assumptions, it is important to have plentiful knowledge of the target characteristics as well as deep understanding of the property of the product or service that one expects to create. These are completely the same as the initial insight when the researcher takes on the studies in various scientific disciplines, and the depth of the initial insight will affect the quality of the research results.

\section{Question2 (Masaaki Mochimaru)}

The expression in your answer, "The process of CCE is designed to readily obtain the insights for designing the product or service, and by setting the initial hypothesis, I think it avoids doing research without a hypothesis and missing out the useful conclusion" is the essence of this paper, and I understand that the way to conduct the research is "one must look at the objective by eliminating any assumptions as much as possible while utilizing the researcher's perspicuity as much as possible". However, 
isn't the "way of doing the research" using the CCE by building the initial hypothesis, hypothesis testing research? Although verification is difficult, for example, if the framework of the product and service are synthesized appropriately, the product or service unseen before is accepted by society or users, and this leads to the change in society, wouldn't this fact be verification that the construction of the framework according to the initial hypothesis and the CCE process was appropriate?

Answer2 (Motoyuki Akamatsu)

I said this was not a hypothesis testing research because the research scenario followed is to conduct the research to the stage of designing the function of the product or service based on the hypothesis (or to determine that it cannot be realized with the current technology), as long as the hypothesis is not wrong without carrying out the strict "verification". Of course, the hypothesis will be proven correct if the functions of the product and service are compatible with the user and as a result, it is accepted by the user or society. However, I would like to state that the objective of the research scenario in the paper is to synthesize (it is "synthesiology") and is not to recognize by hypothesis testing (it is not "epistemology").

In a specific example, in the research of the behavior of the elderly people in train stations, the hypothesis was "there are several different types of impairment of cognitive function in the elderly, and they are impairments of attention or executive functions". When the CCE experiment was planned and conducted based on this hypothesis, it was found that the behaviors in the train station were different according to the types of cognitive function impairment. It can be said that the different types of functional impairment existed, but the objective of the research was not to test the hypothesis that "there are different types of functional impairments", but to propose ways to provide assistance according to functional impairment. (This does not mean that positive proposals could be made. For the group with attention function impairment, the subject could not pay attention no matter how many signs were placed in the station, and it was found that signs were not good ways of assistance.) The research scenario may include the hypothesis testing process, although it is not the objective of the research.

\section{Intervention during behavioral observation and experimental setting \\ Question (Masaaki Mochimaru)}

I think some kind of intervention may be involved in behavioral observation. Unless the intervention is in line with the product or service that will be created, it will not lead to good synthesis even with deep understanding of the behavioral observation with intervention. For example, in the case of service that displays heavily processed, real-time information or service that returns some information by integrating remote information, IT must be used for the intervention, and I think the performance of the experimental IT itself will affect the behavior observation. How should such cases be considered?

\section{Answer (Motoyuki Akamatsu)}

One approach is to create the experiment setting with technological limitations such as remote information as the given condition. For example, if there is a delay in communication, the experiment can be conducted by simulating the delay, if one wants to understand what kind of information provision is pleasing under this condition. How such technological limitation is set as the given condition will differ, for example, depending on whether the researcher wants to use the device or the system as is but wants to improve the contents, or whether he/she can change the performance of the entire device or the system.

In the CCE as a method of synthesiology, I think it is important to set the limiting condition for intervention according to the structure of the product and service to be realized. However, I think we can obtain an innovative product or service that never existed before, if we select the critical parameter for the CCE under no limitations or assumptions as much as possible, rather than setting the structure of the product or service at an early stage and setting them as given.

\section{Quantitative data for product design} Comment (Masaaki Mochimaru)

I think the typical procedure to create a product is to convert the qualitative hypothesis or model obtained by CCE into some sort of quantitative data, and utilizing them in the design of the product or service. Do you have thoughts on how to convert the qualitative model into quantitative data or design?

\section{Answer (Motoyuki Akamatsu)}

The quantitative data needed for product design can be determined by conventional human factor experiments at the stage when the provided contents are determined to some extent by CCE. I think the CCE method will function effectively to discern the preliminary stage for determining the quantitative specification of the product or the requirements of the product and the system. 